\title{
Common features of microRNA target prediction tools
}

\section{Sarah M. Peterson 1,2, Jeffrey A. Thompson ${ }^{3}$, Melanie L. Ufkin $^{1,2}$, Pradeep Sathyanarayana ${ }^{1,2}$, Lucy Liaw ${ }^{1,2}$ and Clare Bates Congdon ${ }^{2,3 *}$}

${ }^{1}$ Center for Molecular Medicine, Maine Medical Center Research Institute, Scarborough, ME, USA

${ }^{2}$ Graduate School of Biomedical Sciences and Engineering, University of Maine, Orono, ME, USA

${ }^{3}$ Department of Computer Science, University of Southern Maine, Portland, ME, USA

\section{Edited by:}

Michael Ochs, The College of New

Jersey, USA

Reviewed by:

Subha Madhavan, Georgetown

University, USA

Ghislain Bidaut, Institut National de

la Santé et de la Recherche

Médicale, France

Madhuchhanda Bhattacharjee,

University of Hyderabad, India

*Correspondence:

Clare Bates Congdon, Department of Computer Science, University of Southern Maine, 96 Falmouth

Street, Portland, ME 04104-9300,

USA

e-mail:congdon@usm.maine.edu
The human genome encodes for over 1800 microRNAs (miRNAs), which are short noncoding RNA molecules that function to regulate gene expression post-transcriptionally. Due to the potential for one miRNA to target multiple gene transcripts, miRNAs are recognized as a major mechanism to regulate gene expression and mRNA translation. Computational prediction of miRNA targets is a critical initial step in identifying miRNA:mRNA target interactions for experimental validation. The available tools for miRNA target prediction encompass a range of different computational approaches, from the modeling of physical interactions to the incorporation of machine learning. This review provides an overview of the major computational approaches to miRNA target prediction. Our discussion highlights three tools for their ease of use, reliance on relatively updated versions of miRBase, and range of capabilities, and these are DIANA-microT-CDS, miRanda-mirSVR, and TargetScan. In comparison across all miRNA target prediction tools, four main aspects of the miRNA:mRNA target interaction emerge as common features on which most target prediction is based: seed match, conservation, free energy, and site accessibility. This review explains these features and identifies how they are incorporated into currently available target prediction tools. MiRNA target prediction is a dynamic field with increasing attention on development of new analysis tools. This review attempts to provide a comprehensive assessment of these tools in a manner that is accessible across disciplines. Understanding the basis of these prediction methodologies will aid in user selection of the appropriate tools and interpretation of the tool output.

Keywords: microRNA, target prediction, seed match, conservation, free energy, site accessibility, machine learning, computational approaches

\section{INTRODUCTION}

MicroRNAs (miRNAs) are $\sim 22$ nucleotide long endogenous RNA regulators of gene activity at the post-transcriptional level. Since the discovery of miRNAs in 1993 (Lee et al., 1993; Wightman et al., 1993), miRNAs have been identified as key regulators of proliferation, differentiation, and cell death in both normal and aberrant pathways (Friedman and Jones, 2009; Garzon et al., 2009; Ambros, 2011; Starega-Roslan et al., 2011; Iuliano et al., 2013). MiRNAs function by targeting complementary sequences in mRNA transcripts, usually in the $3^{\prime}$ untranslated region $\left(3^{\prime}\right.$ UTR), and prevent protein synthesis by inhibiting translation or inducing target degradation. Identification and validation of miRNA:mRNA target interactions is the foundation for discerning the role of miRNAs in the broader context of miRNA regulatory networks governing biological processes.

An extremely large number of potential target sites exists for any given miRNA, and the process of validating a potential miRNA target in the laboratory is time consuming and costly. A computational approach to prediction of miRNA targets facilitates the process of narrowing down potential target sites for experimental validation. Computational approaches model how miRNAs target specific mRNAs and an increasing collection of tools is available, each with a distinct approach to miRNA target prediction. While it may be advantageous to have access to a range of tools with different capabilities, the user is confronted with an important choice in deciding which tool to use.

Although recent reviews exist on human miRNA target identification tools (Reyes-Herrera and Ficarra, 2012; Dweep et al., 2013; Vlachos and Hatzigeorgiou, 2013), this review attempts to present the computational aspects of these tools at a level that is both accurate and accessible across disciplines. Therefore, this review highlights the common features (see Common Features of miRNA Target Prediction Tools) and less common features (see Less Common Features of miRNA Target Prediction Tools) used in developing miRNA target prediction tools, followed by a review of common tools (see Review of Commonly Used miRNA Target Prediction Tools), a summary of excluded tools (see Brief Summary of Tools Excluded from this Review), and lastly a discussion of all of these tools (see Discussion). We have included special consideration of features such as tool maintenance and user-friendliness. We note here the existence of combinations of one or more of these tools into integrated tools. While an evaluation of integrated tools is outside the scope of this review, knowledge of the strengths and limitations of individual component tools is certainly relevant to the user assessment of an integrated tool. Our goal is to provide information for researchers to make an informed decision about which tool to use based on the needs of a particular project. 
COMMON FEATURES OF MIRNA TARGET PREDICTION TOOLS There are four commonly used features for miRNA target prediction tools: seed match, conservation, free energy, and site accessibility. These will be described in the following sections.

\section{SEED MATCH}

The seed sequence of a miRNA is defined as the first 2-8 nucleotides starting at the $5^{\prime}$ end and counting toward the $3^{\prime}$ end (Lewis et al., 2003) (Figure 1). For most tools, a seed match is a Watson-Crick (WC) match between a miRNA and its target in the seed sequence. A WC match between a miRNA and mRNA nucleotide occurs when adenosine (A) pairs with uracil (U) and guanine $(\mathrm{G})$ pairs with cytosine $(\mathrm{C})$. A perfect seed match between the miRNA and the mRNA target has no gaps in alignment within the WC matching.

There are several types of seed matches that can be considered depending on the algorithm. The following types are the main types of seed matches (Lewis et al., 2003, 2005; Brennecke et al., 2005; Krek et al., 2005):

1. 6mer: A perfect WC match between the miRNA seed and mRNA for six nucleotides.

2. 7mer-m8: A perfect WC match from nucleotides $2-8$ of the miRNA seed.

3. 7mer-A1: A perfect WC match from nucleotides $2-7$ of the miRNA seed in addition to an A across from the miRNA nucleotide 1 .

4. 8mer: A perfect WC match from nucleotides 2-8 of the miRNA seed in addition to an A across from the miRNA nucleotide 1.

\section{CONSERVATION}

Conservation refers to the maintenance of a sequence across species. Conservation analysis may focus on regions in the $3^{\prime}$ UTR, the $5^{\prime}$ UTR, the miRNA, or any combination of the three. In general, there is higher conservation in the miRNA seed region than in the non-seed region (Lewis et al., 2003). In a small proportion of miRNA:mRNA target interactions, there is conserved pairing at the $3^{\prime}$ end of the miRNA which can compensate for seed mismatches, and these sites are called $3^{\prime}$ compensatory sites (Friedman et al., 2009). In the context of predicting miRNA targets in $3^{\prime}$ UTRs, conservation analysis may provide evidence that a predicted miRNA target is functional because it is being selected for. Additionally, there is increasing interest in conservation analysis of the genomic regions flanking the miRNA gene and miRNA target genes. As examples, conservation analysis has been applied to the promoter regions of miRNAs and their target genes (Fujiwara and Yada, 2013), and to the co-localization of independently transcribed miRNAs and flanking protein coding genes (Ohler et al., 2004). Thus, the role of conservation in miRNA target prediction is broad and analysis of conserved elements can be incorporated into miRNA target prediction in a variety of ways.

\section{FREE ENERGY}

Free energy (or Gibbs free energy) can be used as a measure of the stability of a biological system. If the binding of a miRNA to a candidate target mRNA is predicted to be stable, it is considered more likely to be a true target of the miRNA. Given the difficulty in measuring free energy directly, usually the change in free energy during a reaction is considered $(\Delta G)$. Since reactions with a negative $\Delta G$ have less energy available to react in the future, they result in systems with increased stability. By predicting how the miRNA and its candidate target hybridize, regions of high and low free energy can be inferred (Figure 2) and the overall $\Delta G$ can be used as an indicator of how strongly bound they are (Yue et al., 2009).

\section{SITE ACCESSIBILITY}

Site accessibility is a measure of the ease with which a miRNA can locate and hybridize with an mRNA target. Following transcription, mRNA assumes a secondary structure (Mahen et al., 2010) which can interfere with a miRNA's ability to bind to a

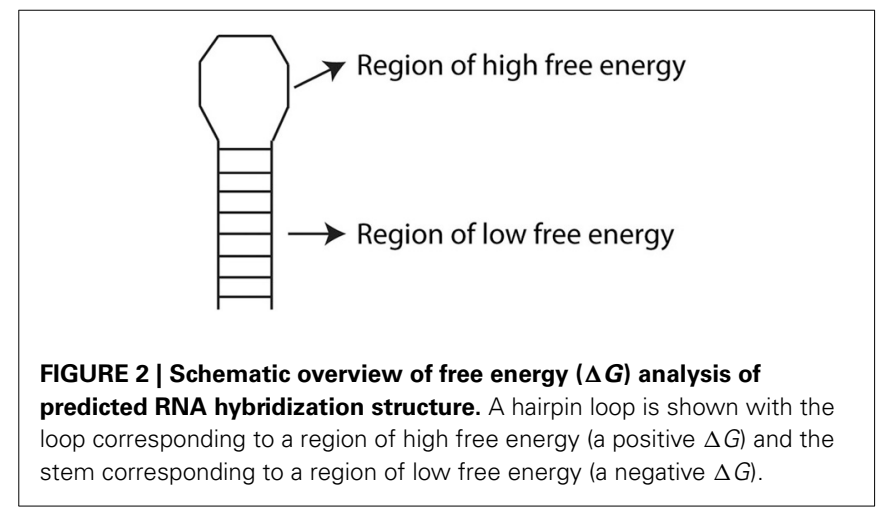

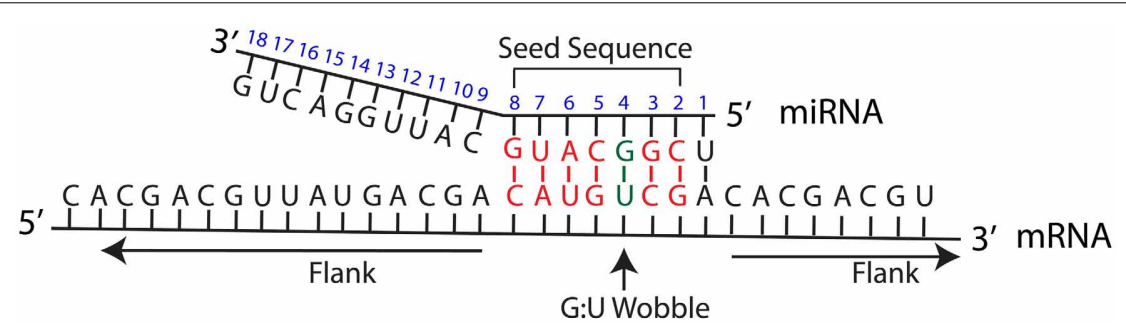

FIGURE 1 | miRNA:mRNA target interaction. Schematic overview of a miRNA interaction with its mRNA target. MiRNA position number is shown in blue. The seed sequence refers to nucleotides in miRNA position number 2-8. Flank refers to the mRNA sequence on either side of the region corresponding to the miRNA seed sequence. WC matches in the seed sequence are shown in red, and an example of $\mathrm{G}-U$ wobble in the seed sequence is shown in green. 
target site. MiRNA:mRNA hybridization involves a two-step process in which a miRNA binds first to a short accessible region of the mRNA. The mRNA secondary structure then unfolds as the miRNA completes binding to a target (Long et al., 2007). Therefore, to assess the likelihood that an mRNA is the target of a miRNA, the predicted amount of energy required to make a site accessible to a miRNA can be evaluated.

\section{LESS COMMON FEATURES OF MIRNA TARGET PREDICTION TOOLS}

The features discussed above are those most commonly incorporated into miRNA target prediction tools. As new advances are made in the characterization of miRNA:mRNA target interactions, additional features are incorporated. These might be used to predict the effectiveness of the target or directly incorporated into the target prediction itself. Target-site abundance is a measure of how many target sites occur in a $3^{\prime}$ UTR (Garcia et al., 2011). Local AU content refers to the concentration of $A$ and $U$ nucleotides flanking the corresponding seed region of the miRNA (Friedman et al., 2009; Betel et al., 2010). GU wobble in the seed match refers to the allowance of a $\mathrm{G}$ pairing with a $\mathrm{U}$ instead of a $\mathrm{C}$ (Doench and Sharp, 2004). 3' compensatory pairing refers to base pair matching with miRNA nucleotides $12-17$. Seed pairing stability is the calculated free energy of the predicted duplex (Garcia et al., 2011). Position contribution analyzes the position of the target site within the mRNA (Grimson et al., 2007). Machinelearning approaches use training data to develop a model of miRNA targets, and then use the model as part of the miRNAprediction process. Machine-learning techniques are likely to use more features in their predictions because they can be trained to determine the predictive power of each feature on positive and negative datasets. A machine-learning approach used by several of these tools is support vector machines (SVM). Tools that use SVM are noted.

\section{REVIEW OF COMMONLY USED MIRNA TARGET PREDICTION TOOLS}

In this section, we outline 10 popular miRNA target prediction tools, using the characteristics previously described. A summary table comparing these tools is provided in the Comparison of miRNA Target Prediction Tools section (Table 11).

\section{miRANDA}

miRanda (Enright et al., 2003) is one of the earlier miRNA target predictors, but it has continued to be updated (Table 1). Although

Table 1 | Profile of miRanda.

\begin{tabular}{ll}
\hline Website & http://www.microrna.org/ (source code) \\
Version & Current version is 3.3a, last updated 8/2010 \\
Input & User-supplied miRNA sequence and UTR sequence \\
for command line & Any \\
Organisms & Free energy threshold, alignment threshold, weight \\
User adjustability & of seed region, and gap penalty \\
Features & Seed match, conservation, and free energy
\end{tabular}

it was originally used to find targets in Drosophila, the algorithm is not limited in this regard and was subsequently used to predict targets in humans (John et al., 2004). Although miRanda is available online as part of the miRanda-mirSVR tool (reviewed below), to be used on its own it must be downloaded.

miRanda uses a three-step analysis. First, the miRNA sequences provided as input are scanned against user-provided $3^{\prime}$ UTRs to check for WC matches. The free energy of each miRNA:mRNA target pair that exceeds a threshold matching score is calculated. Each target that has a predicted free energy below a threshold is then passed to the last step. Finally, conservation is used as a final filter. However, miRanda considers conservation of both binding site and position. The remaining candidates are scored based on how well they match the miRNA. A predicted target can be ranked high in the results by either obtaining a high individual score or by having multiple predicted sites. Unlike most miRNA target predictors, miRanda considers matching along the entire miRNA sequence (Enright et al., 2003). It takes the seed region into account by weighting matches in the seed region more heavily. Matches are allowed to contain limited $\mathrm{G}-\mathrm{U}$ wobble pairs and insertions or deletions (indels). Free energy is calculated by predicting the folding of the miRNA:mRNA hybrid using the Vienna package (Hofacker et al., 1994). Although this is a common method, it ignores any additional protein interaction, such as with the RNA-induced silencing complex (Enright et al., 2003).

miRanda was written in $\mathrm{C}$ and provided as source code. It is relatively easy to compile and run. Nevertheless, both this step and the requirement to run miRanda using the command line will present a technical barrier for many users. However, for more advanced users, miRanda provides a number of adjustable parameters that may be helpful in investigating particular miRNA targets.

\section{miRANDA-mirSVR}

miRanda-mirSVR (Betel et al., 2010) is an online tool that combines two approaches (Table 2). miRanda is used to identify candidate target sites and mirSVR is used to score them. The results are pre-computed, with no option to supply new data. Identification of candidate target sites is described in the section on miRanda. However, scoring is performed using mirSVR, a support vector regression (SVR) approach that is similar to SVM. However, an SVR uses real valued outputs rather than classifying candidates into discrete groups. These are used by mirSVR to compute a score that represents the effect a miRNA may have

\section{Table 2 | Profile of miRanda-mirSVR.}

\begin{tabular}{ll}
\hline Website & http://www.microrna.org/ \\
Version & Current version is 3.3a, last updated 8/2010, uses \\
& miRBase version 15 \\
Input & miRNA identifier or gene name \\
Organisms & Humans, rats, mice, flies, and worms \\
User adjustability & None \\
Features & Seed match, conservation, free energy, site \\
& accessibility, and others
\end{tabular}


Table 3 | Profile of TargetScan.

\begin{tabular}{ll}
\hline Website & http://www.targetscan.org \\
Version & Current version is 6.2, last updated 6/2012 \\
Input & miRNA name, gene name or miRNA family \\
Organism & Mammals, flies, and worms \\
User adjustability & No \\
Features & Seed match and conservation
\end{tabular}

on expression. mirSVR was trained on nine miRNA transfection experiments performed on HeLa cells (Betel et al., 2010) and incorporates a number of other features that it found relevant: site accessibility, AU flanking content, position of the target site within the $3^{\prime}$ UTR, and UTR length.

Although miRanda-mirSVR possesses many of the same capabilities as other prediction tools, the mirSVR score is particularly useful in that it provides an indication of the strength of a miRNA's regulatory effect. Unfortunately, the site is maintained erratically, and does not always use the latest version of miRBase (Kozomara and Griffiths-Jones, 2011) for its predictions. This is a particular problem in terms of using the latest nomenclature when searching for a particular miRNA. Nevertheless, the mirSVR score is a unique and useful capability, and the website is easy to navigate. In addition, the site provides analysis of miRNA expression by tissue and links to miRBase and miRo (The miROntology Database) (Lagana et al., 2009) for more information about a miRNA of interest.

\section{TARGETSCAN}

TargetScan (Lewis et al., 2005; Grimson et al., 2007; Friedman et al., 2009; Garcia et al., 2011) allows the user to search by miRNA name, gene name, or from broadly conserved, conserved, or poorly conserved miRNA families across several species (Table 3 ). The output screen ranks predicted targets by either the predicted efficacy of targeting (context+ scores) or the probability of conserved targeting $\left(\mathrm{P}_{\mathrm{CT}}\right)$. For conservation, the conservation of a $3^{\prime}$ UTR is first determined followed by analysis of a specific k-mer (8mer, 7mer-m8, or 7mer-1A). Since one $3^{\prime}$ UTR can contain multiple target sites, an aggregate $\mathrm{P}_{\mathrm{CT}}$ is provided. For each type of k-mer, the number is provided for that target and whether or not it is considered a conserved site or a poorly conserved site. Furthermore, there is a link to the $3^{\prime}$ UTR of the gene that demonstrates the conserved seed sequence (Friedman et al., 2009). The context+ score demonstrates the probability of a given target as being effectively targeted. Scoring for this feature was derived from experimental results. Several features are included when defining the score, such as $3^{\prime}$ compensatory pairing, local AU content, and position contribution (Grimson et al., 2007; Garcia et al., 2011).

TargetScan is easy to use and actively maintained. It does not require the input of sequences or the adjustment of advanced settings, which could potentially be viewed as an advantage for novice users or a drawback for advanced users.

\section{DIANA-microT-CDS}

DIANA-microT-CDS (Maragkakis et al., 2009; Reczko et al., 2012; Paraskevopoulou et al., 2013) is the latest version of
Table 4 | Profile of DIANA-microT-CDS.

\begin{tabular}{ll}
\hline Website & http://www.microrna.gr/microT-CDS \\
Version & Current version is 5.0, last updated 7/2012, uses \\
& miRBase version 18 \\
miRNA name, gene name, Ensembl ID, KEGG & description, or some combination of these \\
Organisms & Humans, mice, flies, and worms \\
User adjustability & None \\
Features & Seed match, conservation, free energy, site \\
& accessibility and target-site abundance
\end{tabular}

DIANA-microT, which was one of the first miRNA target prediction systems to predict targets in humans (Table 4). The new version incorporates a machine-learning approach to identify the most relevant features extracted from photoactivatableribonucleoside-enhanced crosslinking and immunoprecipitation (PAR-CLIP) data. These data provide DIANA-microT-CDS the opportunity to learn the features associated with miRNA whose binding location is directly known in both coding sequences (CDS) and 3 ' UTR. Additionally, microarray expression data were used to learn the contribution of multiple sites in a target (Reczko et al., 2012). For both regions, the most important features were the binding category weight (as an estimate of the efficiency of binding based primarily on matching in an extended seed sequence), distance to the nearest end of the region (CDS or $3^{\prime}$ UTR), distance to an adjacent binding site, the predicted free energy of the hybrid [using RNAhybrid, reviewed in the RNAhybrid section (Rehmsmeier et al., 2004; Kruger and Rehmsmeier, 2006)], conservation, and AU content. Additionally, the accessibility of the $3^{\prime}$ UTR was found to be relevant, which was predicted using Sfold (Chan et al., 2005). DIANA-microTCDS uses individual models for miRNA binding in both the CDS and the $3^{\prime}$ UTR to separately score targeting in each region before combining both into a single score. Therefore, DIANA-microTCDS is able to predict targeting in cases even when there is no site in the $3^{\prime}$ UTR but at the same time can rank predictions higher that have multiple sites in both regions (Reczko et al., 2012).

DIANA-microT-CDS can be searched by miRNA name, gene name, Ensembl ID, KEGG description, or a combination of these (Paraskevopoulou et al., 2013). Species are specified as part of the miRNA or Ensembl ID, which is somewhat awkward considering that it does not make clear what species are available. However, if it is not specified, DIANA-microT-CDS will ask for clarification. Even with this slight issue, DIANA-microT-CDS is one of the easiest tools to use. The results include the predicted target location, binding type, score, conservation, and links to Ensembl, miRBase, and PubMed that relate to the search (Paraskevopoulou et al., 2013). Additionally, it shows when the target was also predicted by miRanda or TargetScan or was experimentally verified in TarBase (Paraskevopoulou et al., 2013). For advanced users, a Taverna plugin allows more options and a non-web interface.

\section{MirTarget2}

MirTarget2 (Wang, 2008; Wang and El Naqa, 2008) makes miRNA target predictions using SVM and features extracted from a large microarray training dataset (Linsley et al., 2007) (Table 5). This 
Table 5 | Profile of MirTarget2.

\begin{tabular}{ll}
\hline Website & Predicted targets imported into miRDB at \\
http://mirdb.org \\
Version & No version number available, last updated 4/2012 \\
Input & miRNA name, gene name, National Center for \\
& Biotechnology Information (NCBI) RefSeq mRNA \\
& accession number, gene ID or GenBank accession \\
& number \\
Humans, mice, rats, dogs, and chickens \\
User adjustability & $\begin{array}{l}\text { Adjustable and default screening options are available } \\
\text { for the target mining option } \\
\text { Features }\end{array}$ \\
Seed match, conservation, free energy, site \\
accessibility and others (SVM based)
\end{tabular}

machine-learning approach confirmed the use of several popular prediction features and identified new features significantly correlated with miRNA:mRNA target interactions. The training features used include seed conservation, seed match specifically in positions $2-8$, base composition in the regions flanking the seed pairing sites, secondary structure (incorporating site accessibility and free energy), and location of the site within the $3^{\prime}$ UTR. MirTarget 2 was created in conjunction with miRDB (Wang, 2008), and MirTarget2 predictions are available in miRDB. miRDB is a Wikipedia-like functional annotation database for mature miRNA with integration of high throughput automated annotations and manual annotations from individual researchers (Wang, 2008). Seed conservation is incorporated and scored by comparing human, mouse, rat, dog, and chicken orthologs, but is not required. One potential limitation of this program is that the training dataset included only $3^{\prime}$ UTR sequences with a single seed pairing site, as opposed to multiple target sites. The rationale for this was to minimize complications from determining the contribution of each binding site. While this is an understandable choice, it also presents a theoretical limitation of the training dataset given that target-site abundance can alter the likelihood of miRNA:mRNA interactions (Garcia et al., 2011).

Overall, miRDB is actively maintained and user friendly. Predicted miRNA:mRNA target interactions can be searched by miRNA or by mRNA. There is also a target mining option with adjustable and default screening options. This is useful because large numbers of targets are predicted for some miRNAs (e.g., 280 targets for miR-143-3p and 542 targets for miR-145-5p) (Wang, 2008). There are also links to precompiled pathways for miRNA regulators from PANTHER (Protein ANalysis THrough Evolutionary Relationships) (Mi et al., 2013).

\section{rna22-GUI}

rna22-GUI (Loher and Rigoutsos, 2012) is based on rna22 (Hofacker et al., 1994; Miranda et al., 2006), an older target prediction tool (Table 6). rna22 used pattern discovery to identify target islands and evaluate the free energy of paired target islands and candidate miRNAs. Unlike rna22, which required a user-provided miRNA and target sequence, rna22-GUI offers the ability to search by miRNA, gene ID, transcript ID, or gene name. Search results can be presented as a cDNA map, which shows the predicted nucleotide interactions of each of the miRNA:mRNA
Table 6 | Profile of rna22-GUI.

\begin{tabular}{ll}
\hline $\begin{array}{l}\text { Website } \\
\text { Version }\end{array}$ & https://cm.jefferson.edu/rna22v1.0/ \\
& miRBase version 16, Ensembl release 62 \\
miRnA name, Ensembl gene ID, Ensembl transcript & ID, or gene name \\
Organism & Humans, mice, flies, and worms \\
User adjustability & None \\
Features & Seed match and free energy \\
\hline
\end{tabular}

Table 7 | Profile of TargetMiner.

\begin{tabular}{ll}
\hline Website & http://www.isical.ac.in/ bioinfo_miu/targetminer20.htm \\
Version & $\begin{array}{l}\text { No version number available, developed in 2009, } \\
\text { downloadable list of predictions last updated 5/2012 } \\
\text { miRNA name and NCBI RefSeg mRNA accession } \\
\text { number in a user-provided input file }\end{array}$ \\
Organism & Any \\
User adjustability & No \\
Features & Seed match, conservation, free energy, site \\
& accessibility, target-site abundance and others
\end{tabular}

target interaction sites or as a table that lists these predicted duplexes.

While some users may gravitate toward the graphical representation of miRNA:mRNA target interactions, novice users may find the map complicated and difficult to navigate.

\section{TargetMiner}

TargetMiner (Bandyopadhyay and Mitra, 2009) is an SVM-based classifier for identifying potential seed sites between a userprovided miRNA and mRNA of choice (Table 7). The user can search as many miRNAs and targets as desired when uploading the input file. The user is provided with the type of seed match, position, and how many of those sites are found within the sequence. The tool is based on machine learning from negative and positive training data in order to provide more accurate seed match predictions between a miRNA and its target. The positive training data was a set of 289 miRNA transcript pairs extracted from the miRecords database (Xiao et al., 2009). The negative training data was selected from a pooled dataset of pairs of miRNAs and predicted targets by identification of overlapping false positive pairs generated from multiple target prediction algorithms. Tissue specific non-target pairs were then identified by using expression profiling data. While the SVM-based classifier includes consideration of multiple common features inside and outside of the seed region, the output provides the user with information only about the predicted seed match.

Novice users may be dissuaded from using this tool due to the requirement for a preparation of an input file. For advanced users, a downloadable executable version of TargetMiner is available.

\section{SVMicro}

SVMicrO (Liu et al., 2010) is a machine-learning approach to miRNA target prediction (Table 8). The authors used a relatively large positive training data set spanning multiple species. Since 
Table 8 | Profile of SVMicrO.

\begin{tabular}{ll}
\hline Website & http://compgenomics.utsa.edu/svmicro.html \\
Version & No version number available, developed in 2010 \\
Input & User-supplied sequences \\
Organisms & Any \\
User adjustability & None \\
Features & Seed match, conservation, free energy, site \\
& accessibility and target-site abundance
\end{tabular}

they were not able to find experimentally validated negative data, they used expression data. The authors identified 113 possible features of the miRNA binding site, along with 30 possible features of the $3^{\prime}$ UTR as a whole. They ran a minimal redundancy maximal relevance algorithm with the training data to determine which of these features were the best predictors of miRNA regulation. This left them with 21 site-related and 18 UTR-related features, although these features are more granular than those discussed in the Common Features of miRNA Target Prediction Tools section. For example, $8 \mathrm{mer}$ and 7 mer seed matches are considered separate features. SVMicrO uses these features to predict candidate miRNA:mRNA target pairs. Five features (seed match, conservation, free energy, site accessibility, and target-site abundance) were found to be important in predicting miRNA targets, but the training data allowed these to be defined with a tighter focus.

SVMicro's use of numerous granular features in predicting miRNA:mRNA target pairs is powerful. Furthermore, given SVMicrO's relatively large training data set, these features may be useful to other systems. However, usability is currently a limitation. The user needs to build a database containing the UTR to search combined with phastCons conservation scores (Siepel et al., 2005). However, there is no documentation for how this should be done. Furthermore, SVMicrO will only install easily on a 32-bit Linux operating system. It also assumes that the system echo command will be used instead of a built-in shell version. Although it is possible to install it on a 64-bit system, it will be necessary to modify the source. These issues constitute significant obstacles for users unfamiliar with these steps.

\section{PROBABILITY OF INTERACTION BY TARGET ACCESSIBILITY (PITA)}

PITA (Kertesz et al., 2007) uses target-site accessibility as the major feature for miRNA target prediction (Table 9). This is based on the important observation that there is preferential and conserved positioning of target sites in more accessible regions of the UTR. PITA first identifies a potential site by seed match criteria, and then considers site accessibility by computing a free energy score based on the difference between the gain of free energy associated with miRNA:mRNA target duplex formation and the free energy cost of unpairing the target to make it accessible. Next, target-site abundance is considered by combining the site accessibility scores for the same miRNA to identify a total interaction score for the miRNA and UTR. Several options are available for interaction with PITA on the tool website. These include downloading PITA catalogs of predictions and searching predictions by miRNA or by target gene. PITA can also predict which miRNA might target a user-provided UTR sequence. This
Table 9 | Profile of PITA.

\begin{tabular}{|c|c|}
\hline Website & http://genie.weizmann.ac.il/pubs/mir07/ \\
\hline Version & $\begin{array}{l}\text { Current version is } 6 \text {, last updated in } 2008 \text {, uses } \\
\text { miRBase version } 11 \text { and genome versions ce6, } \\
\mathrm{dm} 3, \mathrm{~mm} 9 \text {, and hg18 }\end{array}$ \\
\hline Input & $\begin{array}{l}\text { For the web interface, the user provides miRNA and } \\
\text { gene names or NCBI RefSeq mRNA accession } \\
\text { numbers. A web-based option is available for } \\
\text { user-provided sequence data, and a downloadable } \\
\text { executable version is available }\end{array}$ \\
\hline Organisms & Humans, mice, flies, and worms \\
\hline User adjustability & $\begin{array}{l}\text { Seed size, wobble or mismatch, conservation, and } \\
\text { inclusion of a flank region }\end{array}$ \\
\hline Features & $\begin{array}{l}\text { Seed match, conservation, free energy, site } \\
\text { accessibility and target-site abundance }\end{array}$ \\
\hline
\end{tabular}

\section{Table 10 | Profile of RNAhybrid.}

\begin{tabular}{ll}
\hline Website & http://bibiserv.techfak.uni-bielefeld.de/rnahybrid/ \\
Version & No version number available, developed in 2004 \\
Input & User-supplied data for miRNA sequence and mRNA \\
& sequence \\
Organism & Any \\
User adjustability & Requires advanced user for specification of \\
& parameters \\
Features & Seed match, free energy, and target-site abundance
\end{tabular}

feature is advantageous for an advanced user who wishes to evaluate the $3^{\prime}$ UTR of a novel gene or the $5^{\prime}$ UTR of a gene of interest. With the web version of PITA, users can choose from a selection of pre-set seed match criteria including minimum seed size, allowance of a single G-U wobble or mismatch, minimum seed conservation, and flank settings. Advanced users, however, have the option of downloading the PITA executable with expanded flexibility and advanced parameter setting (Kertesz et al., 2007).

Overall, PITA is a user-friendly tool for both novice and advanced users. While novice users may prefer not to have to enter any seed match parameters, suggested choices for seed parameter settings are provided in the FAQ link. One major limitation of the web version of PITA is that the predictions are based on miRNA sequences from miRBase version 11 (Kertesz et al., 2007). (As of 6/2013, miRBase version 20 has been released, which contains several thousand new entries.) While the reliance on PITA website administrators for continual updates with the latest version of miRBase may be circumvented by downloading the PITA executable, most users are likely to prefer the web-based application.

\section{RNAhybrid}

RNAhybrid (Rehmsmeier et al., 2004; Kruger and Rehmsmeier, 2006) considers the free energy between a miRNA and an mRNA with a user-defined seed region (Table 10). This tool provides a number of advanced settings including specification of hits per target, helix constraints, maximal internal loop size, maximal bulge loop size and maximum free energy cutoff, which are described in detail in the tool manual available at the 
Table 11 | Summary table of miRNA target prediction tools.

\begin{tabular}{|c|c|c|c|c|c|c|c|}
\hline \multicolumn{8}{|c|}{ FEATURES USED IN MIRNA TARGET PREDICTION } \\
\hline Tool name & $\begin{array}{l}\text { Seed } \\
\text { match }\end{array}$ & Conservation & $\begin{array}{c}\text { Free } \\
\text { energy }\end{array}$ & $\begin{array}{l}\text { Site } \\
\text { accessibility }\end{array}$ & $\begin{array}{l}\text { Target-site } \\
\text { abundance }\end{array}$ & $\begin{array}{l}\text { Machine } \\
\text { learning }\end{array}$ & References \\
\hline miRanda-mirSVR & $x$ & $x$ & $x$ & $x$ & & $x$ & Betel et al., 2010 \\
\hline TargetScan & $x$ & $x$ & & & & & $\begin{array}{l}\text { Lewis et al., 2005; } \\
\text { Grimson et al., 2007; } \\
\text { Friedman et al., 2009; } \\
\text { Garcia et al., } 2011\end{array}$ \\
\hline DIANA-microT-CDS & $x$ & $x$ & $x$ & $x$ & $x$ & $x$ & $\begin{array}{l}\text { Maragkakis et al., 2009; } \\
\text { Reczko et al., 2012; } \\
\text { Paraskevopoulou et al., } \\
2013\end{array}$ \\
\hline TargetMiner & $x$ & $x$ & $x$ & $x$ & $x$ & $x$ & $\begin{array}{l}\text { Bandyopadhyay and Mitra, } \\
2009\end{array}$ \\
\hline SVMicrO & $x$ & $x$ & $x$ & $x$ & $x$ & $x$ & Liu et al., 2010 \\
\hline PITA & $x$ & $x$ & $x$ & $x$ & $x$ & & Kertesz et al., 2007 \\
\hline RNAhybrid & $x$ & & $x$ & & $x$ & & $\begin{array}{l}\text { Rehmsmeier et al., 2004; } \\
\text { Kruger and Rehmsmeier, } \\
2006\end{array}$ \\
\hline
\end{tabular}

\begin{tabular}{|c|c|c|c|c|c|c|}
\hline \multicolumn{7}{|c|}{ TOOL AVAILABILITY AND USER FEATURES } \\
\hline Tool name & Website & $\begin{array}{l}\text { Online } \\
\text { use }\end{array}$ & $\begin{array}{l}\text { Source } \\
\text { code }\end{array}$ & $\begin{array}{l}\text { User } \\
\text { adjustability }\end{array}$ & $\begin{array}{l}\text { User-supplied } \\
\text { data required }\end{array}$ & $\begin{array}{l}\text { User } \\
\text { level }\end{array}$ \\
\hline miRanda-mirSVR & http://www.microrna.org/ & $x$ & & & & All \\
\hline TargetScan & http://www.targetscan.org & $x$ & & & & All \\
\hline RNA22-GUI & https://cm.jefferson.edu/rna22v1.0/ & $x$ & & & & Intermediate \\
\hline TargetMiner & $\begin{array}{l}\text { http://www.isical.ac.in/ bioinfo_miu/ } \\
\text { targetminer20.htm }\end{array}$ & $x$ & $x$ & & Input file & Intermediate \\
\hline SVMicrO & $\begin{array}{l}\text { http://compgenomics.utsa.edu/ } \\
\text { svmicro.html }\end{array}$ & $x$ & $x$ & & Sequences & Expert \\
\hline
\end{tabular}

All reviewed tools are freely available for academic use. All tools are actively maintained with updates in the past 5 years, with the exception of PITA and RNAhybrid.

RNAhybrid website. RNAhybrid can also assign a $p$-value for the miRNA:mRNA interaction based on the number of binding sites within the $3^{\prime}$ UTR sequence, which is a measure of target-site abundance.

This tool is intended for advanced users because it requires the input of the miRNA sequence and the mRNA 3' UTR sequence (both in FASTA format) and has options for manipulation of several advanced settings that are specific to this tool.

\section{COMPARISON OF MIRNA TARGET PREDICTION TOOLS}

For ease of comparison, a summary table of reviewed tools is provided (Table 11 ).

\section{BRIEF SUMMARY OF TOOLS EXCLUDED FROM THIS REVIEW}

Space prevents inclusion of an exhaustive listing of miRNA target prediction software, although some of the original miRNA target prediction tools warrant mention, such as Pictar (Krek 
et al., 2005) (based on data that is over 10 years out of date) and rna22 (Hofacker et al., 1994; Miranda et al., 2006) (not functional). Other tools, such as NBmiRTar (Yousef et al., 2007), were excluded based on the use of data that is over 5 years out of date, without an option for the inclusion of updated data. Tools that are not currently operational, such as miTarget (Kim et al., 2006) and MicroInspector (Rusinov et al., 2005), are also excluded. Some tools, such as Genmir++ (Huang et al., 2007) and HuMiTar (Ruan et al., 2008), were excluded on the basis of requiring additional proprietary software and/or expertise for use, which make them inaccessible to the average user. MicroCosm Targets uses the miRanda algorithm and was not separately reviewed (Griffiths-Jones et al., 2008). Space also prevents the review of miRNA target prediction programs exclusively for species other than humans. Lastly, while the emerging field of integrated tools is outside of the scope of this review, elucidation of the strengths and limitations of the component tools is highly relevant to the overall user assessment of an integrated tool.

\section{DISCUSSION}

Identifying the target of a specific miRNA is one approach for discovering the role of the miRNA in normal or aberrant biological processes. Possibly thousands of targets exist, however, for any single miRNA. Over the last 17 years, several tools have been developed to address this complex issue. Each of these projects has contributed to our understanding of the relationship between miRNA and mRNA targets and how that relationship can be used to make accurate predictions.

A recently published study by one of the authors exemplifies how a miRNA target prediction tool can be used to generate candidate targets for subsequent experimental validation (Favreau et al., 2012). While studying the functional role of miR-199b-5p in acute myeloid leukemia (AML), TargetScan was used to examine potential targets of miR-199b-5p based on seed match and conservation. Two highly conserved targets, Podocalyxin (PODXL) and Discoidin Receptor 1 (DDR1), are listed as predicted targets of miR-199b-5p by TargetScan (Garcia et al., 2011; Favreau et al., 2012). Experimental validation via transfection of miR-199b-5p mimics in cell lines confirmed that PODXL and DDR1 are targets of miR-199b-5p at both the mRNA and protein levels (Favreau et al., 2012). Further validation by $3^{\prime}$ UTR luciferase assays confirmed that PODXL and DDR1 are true targets of miR-199b-5p (Favreau et al., 2012).

Although each of the reviewed tools has predictive power, they all have limitations based on the weighting and incorporation of features into the tool. If solely relying on seed match for target identification, a method would exclude whether or not the sequence is conserved or if the site is accessible and thermodynamically favorable. There is evidence that many nonconserved binding sites in the $3^{\prime}$ UTR are functional (Farh et al., 2005). Therefore, relying solely on conservation-based miRNA target prediction systems would be unlikely to capture these miRNA:mRNA interactions (Farh et al., 2005; Witkos et al., 2011). Free energy calculations rely on empiric measurements that may not be complete or accurate (Mathews et al., 1999; Wuchty et al., 1999). The quality of the data used in the free energy calculation can thus be a source of error. Furthermore, relying on a predicted free energy release does not guarantee that the interaction exists. It is important to consider the limitation of each of these common features and how they are used in the context of each tool.

Even though these tools use a combination of features to compensate for the limitations of each feature alone, each tool has its own strengths and limitations. Currently, three of these projects stand out in terms of their wide range of capabilities, ease of use, relatively current input data, and maintenance of the software. These are DIANA-microT-CDS, miRanda-mirSVR, and TargetScan. All of these projects have received periodic updates over the last several years and are easy to use. DIANA-microTCDS uses the most current data out of any of the tools reviewed (miRBase version 18). Furthermore, it is able to make predictions into the CDS in addition to the $3^{\prime}$ UTR. Although miRandamirSVR uses a somewhat older version of miRBase (miRBase version 15) than DIANA-microT-CDS, its mirSVR score is a unique capability that provides a meaningful indication of the degree of regulation. Most other tools only provide a score of the result's significance (which is also provided by miRandamirSVR). In both DIANA-microT-CDS and miRanda-miRSVR, conservation is a feature not a filter, which increases sensitivity to miRNA targets that are lineage specific. TargetScan is based on only slightly older data (miRBase version 17) than DIANAmicroT-CDS. Although it applies a conservation filter, it does allow for poorly conserved targets. Similar to miRanda-mirSVR, it also considers the additional feature of A-U content in the regions flanking the seed region.

Among the remaining target prediction tools reviewed, miRanda is still a widely-used tool even though it needs to be downloaded to be used and it lacks the additional mirSVR score available in miRanda-mirSVR, which may be desirable. rna22-GUI offers a graphical representation of miRNA:mRNA target interactions, but it is based on the original rna22 program and therefore does not incorporate recent advancements in the understanding of miRNA:mRNA target interactions. TargetMiner requires a user-supplied input file and the tool output is limited to seed match characterization. RNAhybrid requires an advanced user due to user-supplied input, adjustment of complex settings, and lack of default values for novice users. The web version of PITA is based on data that is over 5 years out of date, but a downloadable version compatible with user-provided data is available as an alternative option. The final two remaining reviewed tools, SVM-based MirTarget2 and SVMicrO, are machine-learning tools which hold the promise of learning the subtle contributions of many individual features and using them to make more accurate predictions. As more of these features are elucidated and as more positive and negative targets are validated, the promise of machine-learning approaches to use these features to accurately predict targets comes closer to fruition. At present, these last two machine-learning tools do not display a clear advantage over the tools reviewed above and are inherently limited by the lack of extensive positive and negative data training sets available.

In the future, as we gain more understanding of gene regulation and additional predicted miRNA targets are experimentally validated, we expect that current limitations in miRNA target 
prediction tools will be addressed. For example, a method was recently proposed that takes advantage of the observation that a miRNA and its target genes are often co-regulated by common transcription factors, which may eventually be incorporated into new or current target prediction tools (Fujiwara and Yada, 2013). Currently, few of the reviewed target prediction tools are able to address tissue specificity in the prediction of miRNA targets. Tools that allow user-provided data, however, can accommodate some level of tissue specificity by incorporating tissue-specific data such as highly expressed miRNAs or miRNA isoforms, tissue-specific mRNA transcript variants, or lists of highly upregulated or downregulated genes. There is also emerging interest in integrated tools, such as miRmap (Vejnar et al., 2013), that combine multiple miRNA target identification tools to overcome the limitations of individual tools. In addition, some integrated tools, such as MiRonTop (Le Brigand et al., 2010) and CoMiR (Coronnello and Benos, 2013), incorporate expression data in the ranking of miRNA target predictions.

This review highlights the common features of miRNA target prediction and how they are incorporated into different target prediction tools. Further, we encourage the user to be aware of the version, maintenance, and data utilized for each tool. By understanding the features and the tools available, the user is well-equipped to choose the most appropriate miRNA target prediction tool available.

\section{AUTHOR CONTRIBUTIONS}

Sarah M. Peterson, Jeffrey A. Thompson, Melanie L. Ufkin, and Clare Bates Congdon developed the concept for the structure and content of the manuscript. Sarah M. Peterson, Jeffrey A. Thompson, and Melanie L. Ufkin contributed equally to the research and initial draft of the manuscript, with guidance from Clare Bates Congdon. Sarah M. Peterson critically revised the manuscript with assistance from Jeffrey A. Thompson and input from Melanie L. Ufkin, Pradeep Sathyanarayana, Lucy Liaw, and Clare Bates Congdon. All authors reviewed and approved the final version of the manuscript.

\section{ACKNOWLEDGMENTS}

This project was supported in part by a National Institutes of Health COBRE grant in Quantitative Biology (J. Moore, PI; Project \#3: Clare Bates Congdon, PI) from the National Center for Research Resources (5 P20 RR024475-02) and the National Institute of General Medical Sciences (8 P20 GM103534-02) to Clare Bates Congdon and a National Science Foundation CAREER award (\#953495) to Clare Bates Congdon. This project was also supported in part by a National Institutes of Health and National Institute of General Medical Sciences COBRE in Stem Cell Biology and Regenerative Medicine (8P20GM103465, D. Wojchowski, PI; Project \#7: Pradeep Sathyanarayana, PI) to Pradeep Sathyanarayana. The authors would like to thank Dr. Christine Duarte, Senior Biostatistician at Maine Medical Center Research Institute, for critically reviewing this manuscript.

\section{REFERENCES}

Ambros, V. (2011). MicroRNAs and developmental timing. Curr. Opin. Genet. Dev. 21, 511-517. doi: 10.1016/j.gde.2011.04.003
Bandyopadhyay, S., and Mitra, R. (2009). TargetMiner: microRNA target prediction with systematic identification of tissue-specific negative examples. Bioinformatics 25, 2625-2631. doi: 10.1093/bioinformatics/btp503

Betel, D., Koppal, A., Agius, P., Sander, C., and Leslie, C. (2010). Comprehensive modeling of microRNA targets predicts functional non-conserved and noncanonical sites. Genome Biol. 11:R90. doi: 10.1186/gb-2010-11-8-r90

Brennecke, J., Stark, A., Russell, R. B., and Cohen, S. M. (2005). Principles of microRNA-target recognition. PLoS Biol. 3:e85. doi: 10.1371/journal.pbio.0030085

Chan, C. Y., Lawrence, C. E., and Ding, Y. (2005). Structure clustering features on the Sfold Web server. Bioinformatics 21, 3926-3928. doi: 10.1093/bioinformatics/bti632

Coronnello, C., and Benos, P. V. (2013). ComiR: combinatorial microRNA target prediction tool. Nucleic Acids Res. 41, W159-W164. doi: 10.1093/ nar/gkt379

Doench, J. G., and Sharp, P. A. (2004). Specificity of microRNA target selection in translational repression. Genes Dev. 18, 504-511. doi: 10.1101/gad.1184404

Dweep, H., Sticht, C., and Gretz, N. (2013). In-Silico algorithms for the screening of possible microRNA binding sites and their interactions. Curr. Genomics 14, 127-136. doi: 10.2174/1389202911314020005

Enright, A. J., John, B., Gaul, U., Tuschl, T., Sander, C., and Marks, D. S. (2003). MicroRNA targets in drosophila. Genome Biol. 5:R1. doi: 10.1186/gb-20035-1-r1

Farh, K. K., Grimson, A., Jan, C., Lewis, B. P., Johnston, W. K., Lim, L. P., et al. (2005). The widespread impact of mammalian MicroRNAs on mRNA repression and evolution. Science 310, 1817-1821. doi: 10.1126/science. 1121158

Favreau, A. J., Cross, E. L., and Sathyanarayana, P. (2012). miR-199b-5p directly targets PODXL and DDR1 and decreased levels of miR-199b-5p correlate with elevated expressions of PODXL and DDR1 in acute myeloid leukemia. Am. J. Hematol. 87, 442-446. doi: 10.1002/ajh.23129

Friedman, J. M., and Jones, P. A. (2009). MicroRNAs: critical mediators of differentiation, development and disease. Swiss Med. Wkly. 139, 466-472.

Friedman, R. C., Farh, K. K., Burge, C. B., and Bartel, D. P. (2009). Most mammalian mRNAs are conserved targets of microRNAs. Genome Res. 19, 92-105. doi: 10.1101/gr.082701.108

Fujiwara, T., and Yada, T. (2013). miRNA-target prediction based on transcriptional regulation. BMC Genomics 2:S3. doi: 10.1186/1471-2164-14-S2-S3

Garcia, D. M., Baek, D., Shin, C., Bell, G. W., Grimson, A., and Bartel, D. P. (2011). Weak seed-pairing stability and high target-site abundance decrease the proficiency of lsy-6 and other microRNAs. Nat. Struct. Mol. Biol. 18, 1139-1146. doi: $10.1038 / \mathrm{nsmb} .2115$

Garzon, R., Calin, G. A., and Croce, C. M. (2009). MicroRNAs in Cancer. Annu. Rev. Med. 60, 167-179. doi: 10.1146/annurev.med.59.053006.104707

Griffiths-Jones, S., Saini, H. K., van Dongen, S., and Enright, A. J. (2008). miRBase: tools for microRNA genomics. Nucleic Acids Res. 36, D154-D158. doi: 10.1093/nar/gkm952

Grimson, A., Farh, K. K., Johnston, W. K., Garrett-Engele, P., Lim, L. P., and Bartel, D. P. (2007). MicroRNA targeting specificity in mammals: determinants beyond seed pairing. Mol. Cell 27, 91-105. doi: 10.1016/j.molcel.2007.06.017

Hofacker, I. L., Fontana, W., Stadler, P. F., Bonhoeffer, L. S., Tacker, M., and Schuster, P. (1994). Fast folding and comparison of RNA secondary structures. Monatsh. Chem. 125, 167-188. doi: 10.1007/BF00818163

Huang, J. C., Babak, T., Corson, T. W., Chua, G., Khan, S., Gallie, B. L., et al. (2007). Using expression profiling data to identify human microRNA targets. Nat. Methods 4, 1045-1049. doi: 10.1038/nmeth1130

Iuliano, R., Vismara, M. F., Dattilo, V., Trapasso, F., Baudi, F., and Perrotti, N. (2013). The role of microRNAs in cancer susceptibility. Biomed. Res. Int. 2013:591931. doi: 10.1155/2013/591931

John, B., Enright, A. J., Aravin, A., Tuschl, T., Sander, C., and Marks, D. S. (2004). Human MicroRNA targets. PLoS Biol. 2:e363. doi: 10.1371/journal.pbio.0020363

Kertesz, M., Iovino, N., Unnerstall, U., Gaul, U., and Segal, E. (2007). The role of site accessibility in microRNA target recognition. Nat. Genet. 39, 1278-1284. doi: $10.1038 /$ ng2 135

Kim, S. K., Nam, J. W., Rhee, J. K., Lee, W. J., and Zhang, B. T. (2006). miTarget: microRNA target gene prediction using a support vector machine. BMC Bioinformatics 7:411. doi: 10.1186/1471-2105-7-411 
Kozomara, A., and Griffiths-Jones, S. (2011). miRBase: integrating microRNA annotation and deep-sequencing data. Nucleic. Acids Res. 39, D152-D157. doi: 10.1093/nar/gkq1027

Krek, A., Grun, D., Poy, M. N., Wolf, R., Rosenberg, L., Epstein, E. J., et al. (2005). Combinatorial microRNA target predictions. Nat. Genet. 37, 495-500. doi: $10.1038 /$ ng 1536

Kruger, J., and Rehmsmeier, M. (2006). RNAhybrid: microRNA target prediction easy, fast and flexible. Nucleic Acids Res. 34, W451-W454. doi: 10.1093/nar/gkl243

Lagana, A., Forte, S., Giudice, A., Arena, M. R., Puglisi, P. L., Giugno, R., et al. (2009). miRo: a miRNA knowledge base. Database (Oxford) 2009, bap008. doi: 10.1093/database/bap008

Le Brigand, K., Robbe-Sermesant, K., Mari, B., and Barbry, P. (2010). MiRonTop: mining microRNAs targets across large scale gene expression studies. Bioinformatics 26, 3131-3132. doi: 10.1093/bioinformatics/btq589

Lee, R. C., Feinbaum, R. L., and Ambros, V. (1993). The C. elegans heterochronic gene lin-4 encodes small RNAs with antisense complementarity to lin-14. Cell 75, 843-854. doi: 10.1016/0092-8674(93)90529-Y

Lewis, B. P., Burge, C. B., and Bartel, D. P. (2005). Conserved seed pairing, often flanked by adenosines, indicates that thousands of human genes are microRNA targets. Cell 120, 15-20. doi: 10.1016/j.cell.2004.12.035

Lewis, B. P., Shih, I. H., Jones-Rhoades, M. W., Bartel, D. P., and Burge, C. B. (2003). Prediction of mammalian microRNA targets. Cell 115, 787-798. doi: 10.1016/S0092-8674(03)01018-3

Linsley, P. S., Schelter, J., Burchard, J., Kibukawa, M., Martin, M. M., Bartz, S. R., et al. (2007). Transcripts targeted by the microRNA-16 family cooperatively regulate cell cycle progression. Mol. Cell. Biol. 27, 2240-2252. doi: 10.1128/MCB.02005-06

Liu, H., Yue, D., Chen, Y., Gao, S. J., and Huang, Y. (2010). Improving performance of mammalian microRNA target prediction. BMC Bioinformatics 11:476. doi: 10.1186/1471-2105-11-476

Loher, P., and Rigoutsos, I. (2012). Interactive exploration of RNA22 microRNA target predictions. Bioinformatics 28, 3322-3323. doi: 10.1093/bioinformatics/bts615

Long, D., Lee, R., Williams, P., Chan, C. Y., Ambros, V., and Ding, Y. (2007). Potent effect of target structure on microRNA function. Nat. Struct. Mol. Biol. 14, 287-294. doi: 10.1038/nsmb1226

Mahen, E. M., Watson, P. Y., Cottrell, J. W., and Fedor, M. J. (2010). mRNA secondary structures fold sequentially but exchange rapidly in vivo. PLoS Biol. 8:e1000307. doi: 10.1371/journal.pbio.1000307

Maragkakis, M., Reczko, M., Simossis, V. A., Alexiou, P., Papadopoulos, G. L., Dalamagas, T., et al. (2009). DIANA-microT web server: elucidating microRNA functions through target prediction. Nucleic Acids Res. 37, W273-W276. doi: 10.1093/nar/gkp292

Mathews, D. H., Sabina, J., Zuker, M., and Turner, D. H. (1999). Expanded sequence dependence of thermodynamic parameters improves prediction of RNA secondary structure. J. Mol. Biol. 288, 911-940. doi: 10.1006/jmbi.1999.2700

Mi, H., Muruganujan, A., and Thomas, P. D. (2013). PANTHER in 2013: modeling the evolution of gene function, and other gene attributes, in the context of phylogenetic trees. Nucleic Acids Res. 41, D377-D386. doi: 10.1093/nar/gks1118

Miranda, K. C., Huynh, T., Tay, Y., Ang, Y. S., Tam, W. L., Thomson, A. M., et al. (2006). A pattern-based method for the identification of MicroRNA binding sites and their corresponding heteroduplexes. Cell 126, 1203-1217. doi: 10.1016/j.cell.2006.07.031

Ohler, U., Yekta, S., Lim, L. P., Bartel, D. P., and Burge, C. B. (2004). Patterns of flanking sequence conservation and a characteristic upstream motif for microRNA gene identification. RNA 10, 1309-1322. doi: 10.1261/rna.5206304

Paraskevopoulou, M. D., Georgakilas, G., Kostoulas, N., Vlachos, I. S., Vergoulis, T., Reczko, M., et al. (2013). DIANA-microT web server v5.0: service integration into miRNA functional analysis workflows. Nucleic Acids Res. 41, W169-W173. doi: 10.1093/nar/gkt393

Reczko, M., Maragkakis, M., Alexiou, P., Grosse, I., and Hatzigeorgiou, A. G. (2012). Functional microRNA targets in protein coding sequences. Bioinformatics 28, 771-776. doi: 10.1093/bioinformatics/bts043
Rehmsmeier, M., Steffen, P., Hochsmann, M., and Giegerich, R. (2004). Fast and effective prediction of microRNA/target duplexes. RNA 10, 1507-1517. doi: 10.1261/rna.5248604

Reyes-Herrera, P. H., and Ficarra, E. (2012). One decade of development and evolution of microRNA target prediction algorithms. Genomics Proteomics Bioinformatics 10, 254-263. doi: 10.1016/j.gpb.2012.10.001

Ruan, J., Chen, H., Kurgan, L., Chen, K., Kang, C., and Pu, P. (2008). HuMiTar: a sequence-based method for prediction of human microRNA targets. Algorithms Mol. Biol. 3:16. doi: 10.1186/1748-7188-3-16

Rusinov, V., Baev, V., Minkov, I. N., and Tabler, M. (2005). MicroInspector: a web tool for detection of miRNA binding sites in an RNA sequence. Nucleic Acids Res. 33, W696-W700. doi: 10.1093/nar/gki364

Siepel, A., Bejerano, G., Pedersen, J. S., Hinrichs, A. S., Hou, M., Rosenbloom, K., et al. (2005). Evolutionarily conserved elements in vertebrate, insect, worm, and yeast genomes. Genome Res. 15, 1034-1050. doi: 10.1101/gr.3715005

Starega-Roslan, J., Koscianska, E., Kozlowski, P., and Krzyzosiak, W. J. (2011). The role of the precursor structure in the biogenesis of microRNA. Cell. Mol. Life Sci. 68, 2859-2871. doi: 10.1007/s00018-011-0726-2

Vejnar, C. E., Blum, M., and Zdobnov, E. M. (2013). miRmap web: comprehensive microRNA target prediction online. Nucleic Acids Res. 41, W165-W168. doi: $10.1093 /$ nar/gkt430

Vlachos, I. S., and Hatzigeorgiou, A. G. (2013). Online resources for miRNA analysis. Clin. Biochem. 46, 879-900. doi: 10.1016/j.clinbiochem.2013.03.006

Wang, X. (2008). miRDB: a microRNA target prediction and functional annotation database with a wiki interface. RNA 14, 1012-1017. doi: 10.1261/rna.965408

Wang, X., and El Naqa, I. M. (2008). Prediction of both conserved and nonconserved microRNA targets in animals. Bioinformatics 24, 325-332. doi: 10.1093/bioinformatics/btm595

Wightman, B., Ha, I., and Ruvkun, G. (1993). Posttranscriptional regulation of the heterochronic gene lin-14 by lin-4 mediates temporal pattern formation in $C$. elegans. Cell 75, 855-862. doi: 10.1016/0092-8674(93)90530-4

Witkos, T. M., Koscianska, E., and Krzyzosiak, W. J. (2011). Practical aspects of microRNA target prediction. Curr. Mol. Med. 11, 93-109. doi: 10.3791/2034

Wuchty, S., Fontana, W., Hofacker, I. L., and Schuster, P. (1999). Complete suboptimal folding of RNA and the stability of secondary structures. Biopolymers 49, 145-165. doi: 10.1002/(SICI)1097-0282(199902)49:2<145::AID-BIP4>3.0. $\mathrm{CO} ; 2-\mathrm{G}$

Xiao, F., Zuo, Z., Cai, G., Kang, S., Gao, X., and Li, T. (2009). miRecords: an integrated resource for microRNA-target interactions. Nucleic Acids Res. 37, D105-D110. doi: 10.1093/nar/gkn851

Yousef, M., Jung, S., Kossenkov, A. V., Showe, L. C., and Showe, M. K. (2007). Naive Bayes for microRNA target predictions-machine learning for microRNA targets. Bioinformatics 23, 2987-2992. doi: 10.1093/bioinformatics/btm 484

Yue, D., Liu, H., and Huang, Y. (2009). Survey of computational algorithms for MicroRNA target prediction. Curr. Genomics 10, 478-492. doi: $10.2174 / 138920209789208219$

Conflict of Interest Statement: The authors declare that the research was conducted in the absence of any commercial or financial relationships that could be construed as a potential conflict of interest.

Received: 22 November 2013; accepted: 23 January 2014; published online: 18 February 2014.

Citation: Peterson SM, Thompson JA, Ufkin ML, Sathyanarayana P, Liaw L and Congdon CB (2014) Common features of microRNA target prediction tools. Front. Genet. 5:23. doi: 10.3389/fgene.2014.00023

This article was submitted to Bioinformatics and Computational Biology, a section of the journal Frontiers in Genetics.

Copyright (C) 2014 Peterson, Thompson, Ufkin, Sathyanarayana, Liaw and Congdon. This is an open-access article distributed under the terms of the Creative Commons Attribution License (CC BY). The use, distribution or reproduction in other forums is permitted, provided the original author(s) or licensor are credited and that the original publication in this journal is cited, in accordance with accepted academic practice. No use, distribution or reproduction is permitted which does not comply with these terms. 